\title{
BMJ Open Opportunistic HPV vaccination at age 16-23 and cervical screening attendance in Sweden: a national register-based cohort study
}

Teresa Kreusch, ${ }^{1}$ Jiangrong Wang, ${ }^{1}$ Pär Sparén, ${ }^{1}$ Karin Sundström ${ }^{2,3}$

To cite: Kreusch T, Wang J, Sparén P, et al. Opportunistic $\mathrm{HPV}$ vaccination at age 16-23 and cervical screening attendance in Sweden: a national register-based cohort study. BMJ Open 2018;8:e024477. doi:10.1136/ bmjopen-2018-024477

- Prepublication history and additional material for this paper are available online. To view these files, please visit the journal online (http://dx.doi org/10.1136/bmjopen-2018024477).

Received 28 May 2018 Revised 8 August 2018 Accepted 27 August 2018

\section{Check for updates}

C C Author(s) (or their employer(s)) 2018. Re-use permitted under CC BY-NC. No commercial re-use. See rights and permissions. Published by BMJ.

${ }^{1}$ Department of Medical Epidemiology and Biostatistics, Karolinska Institutet, Stockholm, Sweden

${ }^{2}$ Department of Laboratory Medicine, Karolinska Institutet, Stockholm, Sweden

${ }^{3}$ Center for Cervical Cancer

Prevention, Karolinska University Laboratory, Karolinska University Hospital, Stockholm, Sweden

Correspondence to Dr Karin Sundström; karin.sundstrom@ki.se

\section{ABSTRACT}

Objectives To investigate whether cervical screening attendance differs between human papillomavirus (HPV)vaccinated and unvaccinated women and to investigate potential underlying socioeconomic factors.

Design Prospective cohort using registry linkage of vaccinations, screening invitations, screening attendance and socioeconomic covariates.

Setting Swedish national HPV vaccination and cervical screening programmes.

Participants All Swedish women born between 1988 and 1991 and invited to screening ( $\mathrm{n}=261434)$.

Outcome measures All participants were followed for up to 3 years. Screening attendance was compared between HPV-vaccinated and unvaccinated women. $\mathrm{HR}$ and $95 \% \mathrm{Cl}$ were estimated using Cox regression.

Results Vaccination age averaged 18.1 years and the coverage for $\geq 1$ dose was $13.5 \%$. In HPV-vaccinated women ( $n=35460)$, screening attendance was higher than in unvaccinated women $(\mathrm{n}=225974)(74 \% \mathrm{vs} 69 \%$, $\mathrm{p}<0.001)$. The crude HR of attendance in HPV-vaccinated women was 1.32 (95\% $\mathrm{Cl} 1.30$ to 1.34). A positive association remained after adjustment for education, income and migration history $(\mathrm{HR}=1.10,95 \% \mathrm{Cl} 1.09$ to 1.12).

Conclusion HPV-vaccinated women were more likely to attend screening than unvaccinated women. Yet, the question needs to be reassessed in routinely vaccinated cohorts, since the vaccinated women included here represent a selected group and may be prone to more health-conscious habits.

\section{INTRODUCTION}

Human papillomavirus (HPV) vaccines do not provide complete protection against all oncogenic HPV types; hence, cervical screening remains essential in HPV-vaccinated women. ${ }^{12}$ Sweden approved the quadrivalent HPV vaccine in October 2006 and organised vaccination programmes began in June 2012. In the interim period, a smaller proportion of Swedish girls aged 13-17 years were vaccinated opportunistically, that is, in a non-organised manner. The cost of approximately $€ 330$ for three doses was partially or
Strengths and limitations of this study

- This study used register data with very high completeness and individual linkage to socioeconomic covariates. The overall level of differential misclassification and bias in this study is minimal and the internal validity is high.

- This means the generalisability to current vaccinated cohorts is excellent.

- Our chief limitation is that the participants still were not part of an organised school-based programme, and thus self-selected (on their own and/or parental volition) whether to be vaccinated or not.

- The latter limitation entails that generalisability to future vaccinated cohorts is uncertain-further follow-up is needed of these.

wholly subsidised, depending on the family's medical expense level, as part of a national pharmaceutical benefits scheme. On average, the cost for three doses of the vaccine was reduced to around half by this scheme. ${ }^{34}$ The subsidy was later extended to women up to the age of 26. These girls and young women were however vaccinated later than at the recommended age of 10-12 years, ${ }^{5}$ meaning that many had likely already been exposed to oncogenic HPV types. ${ }^{6}$ Given the vaccine's inability to cure ongoing infections, ${ }^{7}$ the potentially weaker vaccine-induced antibody response in 16-23-year-olds compared with younger girls ${ }^{8}$ and the decrease in effectiveness with increasing age at vaccination, ${ }^{9}{ }^{10}$ continued cervical screening is crucial especially in these women. In Sweden, invitations to cervical screening are issued every 3 years for women aged 23-50, every 5 years for women aged 51-64 and to every woman migrating to Sweden. ${ }^{11} 12$ Eighty-two per cent of Swedish women attend at least one cervical screening within the recommended time interval. ${ }^{11}$ Women who did not attend are reinvited after 1 year. ${ }^{11}$ 
Some concerns about decreasing screening attendance were voiced on the HPV vaccine's introduction, supposing that vaccinated women might perceive a false sense of full protection and therefore refrain from screening. ${ }^{13-15}$ However, only one study has so far reported decreased screening attendance in HPV-vaccinated women, ${ }^{16}$ several others suggest that vaccinated women screen equally or more often than unvaccinated women. ${ }^{17-24}$

This was also found in a Swedish study on birth cohorts $1977-1987 .^{18}$ In that study, vaccinations occurred entirely at an out-of-pocket price of $€ 330$ with a strong self-selection of women. ${ }^{18}$ Moreover, vaccine uptake was only $0.8 \%$ in the assessed birth cohorts, ${ }^{18}$ resulting in limited generalisability of findings. The present study aims to reassess the association between HPV vaccination and cervical screening in the birth cohorts 1988-1991, who were younger at vaccination and had higher vaccination uptake levels of up to $29 \%,{ }^{5}$ as part of our continuous follow-up of HPV vaccination in Sweden.

\section{METHODS}

\section{Study population}

In this national register-based cohort study, the study population included all women registered in Sweden who were born between 1988 and 1991, and were invited to cervical screening (figure 1). Women who emigrated or died prior to the study period or who did not receive a screening invitation were excluded. The non-invited women were subject to a separate descriptive analysis. Moreover, women were excluded if they had screened on their own initiative before being invited. The genetic component in cervical cancer may influence the sensitivity to persistent HPV infections ${ }^{25}$ and it is possible that more early screeners, for example, had a family history of cervical pathology. They may also have been more likely to have symptoms of bleeding and/or condyloma, which would render them unrepresentative of the main study population. Early screeners, that is, those screening before invitation, were therefore also subject to a separate descriptive analysis.

Cohort entry was defined as date of the first screening invitation after 1 January 2010, that is, when the first women in the study turned 22-23 and thus were eligible to start organised screening. Women were followed until attending screening, or until censored at emigration, death, 3years after invitation (ie, one screening interval) or the end of the study period on 31 December 2015, whichever occurred first.

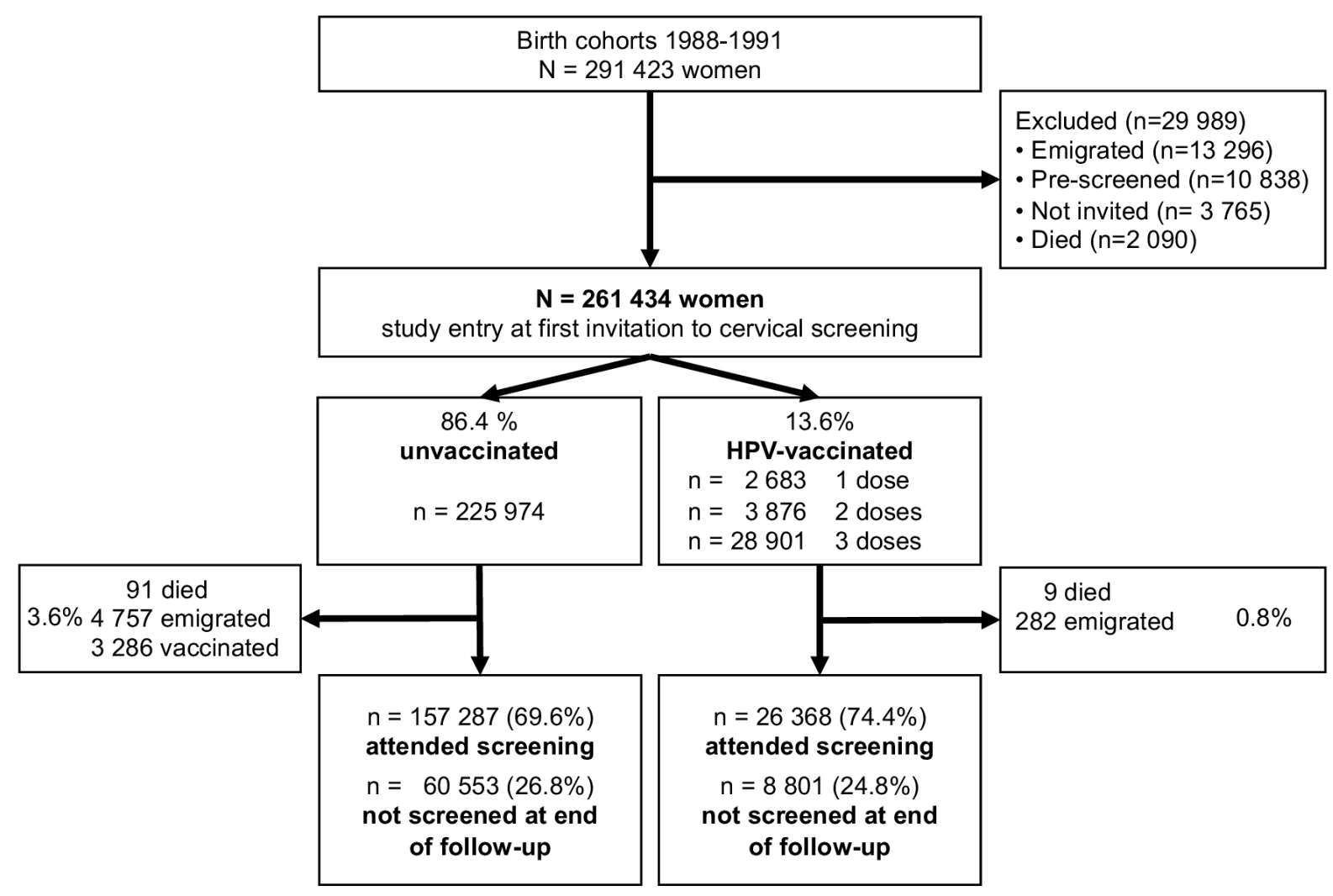

Figure 1 Composition of the register-derived study cohort. 


\section{Data sources and definition of variables}

Initially, records of all women born in 1988-1991 were extracted from Sweden's Total Population Register. Individual-level data from other population-based registers were then cross-linked based on unique personal identification numbers and pseudonymised before analysis. Dates of death and migration were retrieved from the Causes of Death Register and the Migration Register, respectively.

Dates of screening invitations, that is, the point of study entry, and all organised and opportunistic smears, that is, the outcome events, were retrieved from the Swedish National Cervical Screening Registry (NKCx as Swedish acronym), which is $100 \%$ complete since $1995 .{ }^{11} \mathrm{HPV}$ vaccination was defined as receipt of at least one dose of the bivalent or quadrivalent HPV vaccine before study entry (A one doseonly vaccine regimen is not currently recommended, yet for this study, we hypothesised that one dose might already suffice to be associated with differing screening uptake, whether positive or negative). Women who were vaccinated after study entry were censored. An additional sensitivity analysis instead treated HPV vaccination as a time-varying exposure. Vaccination data originated from the SVEVAC register, which covers $85 \%-90 \%$ of vaccines administered in Sweden ${ }^{5}$ and from the Prescribed Drug Register (PDR), which captures all medical prescriptions and is complete since $2005 .{ }^{26}$ Participants were considered to have benefitted from the subsidy when their first vaccination occurred after 9 May 2007 for women then aged 13-17 and after 28 June 2012 for women up to age $26{ }^{4}$

The Database for Health Insurance and Labour Market Studies (LISA as Swedish acronym) ${ }^{27}$ provided data on education level at study entry of individuals and their biological or adoptive parents. Linkage to parents' records was performed using the Multi-Generation Register (MGR). Education was categorised into compulsory school ( $\leq 9$ years), high school (9-12years) and university and above ( $\geq 12$ years). LISA also provided data on disposable income, measured at study entry in Swedish kronor (SEK) per year. The quartile cut-offs were 93 100, 136900 and 183500 SEK for individual income and 128000,193700 and 387800 SEK for family income. Family history of abnormal cytological screening results and histological-confirmed CIN2+ lesionswas obtained for all first-degree female relatives from the NKCx, as linked via MGR.

\section{Statistical analysis}

We compared the cumulative incidence proportion of screening attendance after invitation between HPV-vaccinated and unvaccinated women, using Kaplan-Meier function. HR with $95 \%$ CI of screening attendance among the HPV-vaccinated compared with the unvaccinated were estimated using Cox regression, using attained age as the underlying time scale, as previously described. ${ }^{18}$

Using explorative model building, potential confounding factors were added to the crude model. Only covariates that noticeably affected the main HR estimate by at least one decimal place and improved model fit were retained in the main effects model (individual education, individual income, migration history); others were dropped (family income, parents' education, family history of cervical lesions). For the fully adjusted model, see online supplementary 1 . Interaction effects were explored between vaccination and education based on previous research ${ }^{18} 28$ and also between vaccination and income and vaccination and migration history, since the high vaccine cost might have led to differential effects depending on income quartile or migration status. Stratified analyses were performed to explore the association by vaccine dose number. The Akaike information criterion was used to determine the best-fitting model. All tests of significance were two-sided and $p$ value of 0.05 was considered statistically significant. No violations of the proportional hazards assumption were detected when visually inspecting scaled Schoenfeld residuals. ${ }^{29}$

\section{Sensitivity analyses}

To investigate if our results were robust, vaccination status was first redefined as having received the full three vaccine doses. Second, only women invited to screening at ages 22-24 were included, as the women who received invitations at older ages might differ in characteristics, for instance, in migration history. Third, vaccination was redefined as a time-varying exposure.

Data linkage and management was performed with SAS V9.4 software (SAS Institute, Cary, North Carolina, USA). Statistical analyses were conducted in Stata V.14 software (Stata Corp, Texas, USA).

\section{Patient involvement}

No patients were involved in setting the research question or the outcome measures, nor were they involved in developing plans for design or implementation of the study. No patient was asked to advise on interpretation or writing up of results. There are no plans to disseminate the results of the research to study participants or the relevant patient community.

\section{RESULTS}

\section{Characteristics of the study population}

The final cohort comprised 261434 women (figure 1): $13.6 \%(\mathrm{n}=35460)$ were HPV-vaccinated with at least one dose and most had received all three doses. HPV-vaccinated women were more likely to have university education, to be in the upper income quartile and to be born in Sweden (table 1).

Vaccination coverage increased by birth year, with $3.6 \%$ among women born in 1988, 6.7\% in those born in 1989, $15.3 \%$ in those born in 1990 and $28.6 \%$ in those born in 1991. Vaccinations occurred at a mean age of 18.1 years ( $\mathrm{SD}=1.9$ years) and $75.1 \%$ of vaccinations were eligible for the subsidy (see online supplementary 2).

The descriptive analysis on the excluded women showed that those who had screened on their own 
Table 1 Characteristics of the study cohort of women born 1988-1991

\begin{tabular}{|c|c|c|c|}
\hline & Unvaccinated & Vaccinated & Total \\
\hline & n (\%) & $\mathrm{n}(\%)$ & n (\%) \\
\hline Total cohort & $225974(100.0)$ & $35460(100.0)$ & 261434 \\
\hline Age at screening invitation (years) & 23.3 (SD 0.8) & $23.2(\mathrm{SD} 0.6)$ & 23.3 (SD 0.8) \\
\hline \multicolumn{4}{|l|}{ Follow-up time (person-years) } \\
\hline Total follow-up time & 230945 & 25371 & 256316 \\
\hline Mean follow-up time & $1.02(\mathrm{SD} 1.03)$ & 0.72 (SD 0.79) & 0.98 (SD 1.01) \\
\hline \multicolumn{4}{|l|}{ Screening attendance } \\
\hline Attended screening & $157287(69.6)$ & $26368(74.4)$ & $183655(70.2)$ \\
\hline Did not attend screening & $68687(30.4)$ & $9092(25.6)$ & $77779(29.8)$ \\
\hline \multicolumn{4}{|l|}{ Education } \\
\hline Compulsory school (<9years) & $20927(9.3)$ & $1049(3.0)$ & $21976(8.4)$ \\
\hline High school (9-12 years) & $100090(44.3)$ & $12933(36.5)$ & $113023(43.2)$ \\
\hline University (>12 years) & 81049 (35.9) & $21191(59.8)$ & $102240(39.1)$ \\
\hline Missing & 23908 (10.6) & $287(0.8)$ & $24195(9.3)$ \\
\hline \multicolumn{4}{|l|}{ Individual income } \\
\hline Quartile 1 & $59570(26.4)$ & $4563(12.9)$ & $64133(24.5)$ \\
\hline Quartile 2 & $56027(24.8)$ & $8101(22.9)$ & $64128(24.5)$ \\
\hline Quartile 3 & $54052(23.9)$ & $10077(28.4)$ & $64129(24.5)$ \\
\hline Quartile 4 & $51380(22.7)$ & $12716(35.9)$ & $64096(24.5)$ \\
\hline Missing & $4945(2.2)$ & $3(0.0)$ & 4948 (1.9) \\
\hline \multicolumn{4}{|l|}{ Migration history } \\
\hline Born in Sweden & $178847(79.1)$ & $33924(95.7)$ & 212771 (81.4) \\
\hline Immigrated $\geq 10$ years ago & $14251(6.3)$ & 1175 (3.3) & $15426(5.9)$ \\
\hline Immigrated $<10$ years ago & 32876 (14.6) & $361(1.0)$ & 33237 (12.7) \\
\hline
\end{tabular}

All differences between exposure groups, as assessed with t-tests and $\chi^{2}$ tests, were statistically significant at $p<0.001$, as expected given the large sample size.

Vaccinated, human papillomavirus vaccinated with $\geq 1$ dose; SD, standard deviation.

initiative before being invited ( $\mathrm{n}=10838)$ had a higher vaccination coverage at $22.4 \%$. A family history of cervical lesions was present in $30.8 \%$ of this group, compared with $23.2 \%$ in the main cohort. The overall proportion of early screeners $(3.7 \%)$ was similar to that among the preceding 10 birth cohorts $(3.6 \%){ }^{18}$ Of those who had not been invited to screening $(\mathrm{n}=3765), 57 \%$ were recent immigrants and might therefore not have been invited yet. Overall, the lack of invitation remained unexplained for 1637 women. Over half of these also lacked basic demographic data such as birth country and might therefore not have been invited.

\section{Screening invitations and attendance}

Age at screening invitation was similar in vaccinated and unvaccinated women (table 1). A minority of women $(5.6 \%)$ were invited above age 24 and more than half of these had a history of migration. The crude screening attendance, defined as the proportion of being screened up to 3 years after invitation, among all women was $70.2 \%$. When stratified by vaccination status, this attendance was higher in the vaccinated, at $74.4 \%$, compared with the unvaccinated, at $69.6 \%$ $(p<0.001)$. This pattern emerged early on, remained stable throughout follow-up and was statistically significant (log-rank test $\mathrm{p}<0.001)$ (figure 2$)$.

Table 2 displays the crude and adjusted HR for screening attendance in vaccinated compared with unvaccinated women. In the crude model, vaccinated women were $32 \%$ more likely to attend screening. The difference decreased to $10 \%$ in the adjusted model, but remained statistically significant. When separating the vaccinated group by women's eligibility for the subsidy, results remained similar but with higher attendance among women who paid the full vaccine cost themselves (see online supplementary 3 ).

The analysis by vaccine dose yielded a distribution of $7.6 \%$ for one dose, $10.9 \%$ for two doses and $81.5 \%$ for three doses among vaccinated women ( $\mathrm{p}$ for trend $<0.001)$. Compared with unvaccinated women, the association between vaccination and screening was positive in all strata and the overall difference in attendance was largest in the three-dose stratum (table 2). 


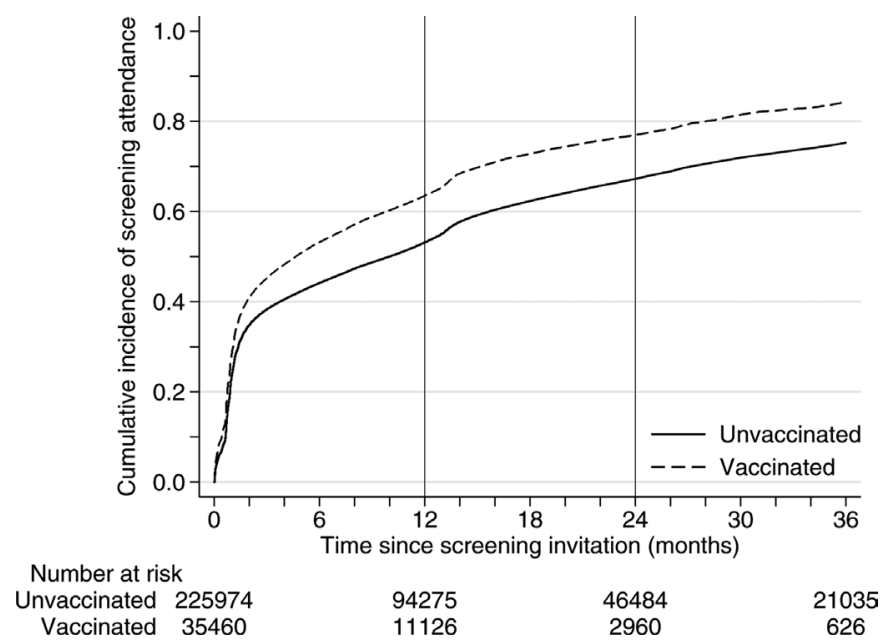

Figure 2 Cumulative incidence proportion of cervical screening attendance after invitation in women born 19881991, by human papillomavirus vaccination status.

All three tested interactions (table 3 ) were statistically significant (likelihood ratio test $\mathrm{p}<0.001$ for each) and separate inclusion of each interaction term improved model fit. Attendance differed most noticeably between vaccinated and unvaccinated women in the lowest income quartile, at $22 \%$, and least in the highest quartile, at $3 \%$. Similarly, the association was stronger in women who had immigrated to Sweden than in those born there. Regarding education, the association in women with university education is slightly higher than that in women in the other categories (table 3 ).

Table 2 Crude model, adjusted model with main effects, and model with the exposure split to three levels on the association between HPV vaccination and cervical screening attendance $(n=261434)$

\begin{tabular}{|c|c|c|c|}
\hline Model & $\begin{array}{l}\text { Exposure } \\
\text { status }\end{array}$ & $\begin{array}{l}\text { Crude } \\
\text { attendance n } \\
(\%)\end{array}$ & HR $(95 \% \mathrm{Cl})$ \\
\hline \multicolumn{4}{|c|}{ Crude model } \\
\hline & Unvac. & $157287(69.6)$ & ref. \\
\hline & Vac. & $26368(74.4)$ & $1.32(1.30$ to 1.34$)$ \\
\hline \multicolumn{4}{|c|}{ Adjusted model with main effects* } \\
\hline & Unvac. & $157287(69.6)$ & ref. \\
\hline & Vac. & $26368(74.4)$ & $1.10(1.09$ to 1.12$)$ \\
\hline \multicolumn{4}{|c|}{ Exposure split by number of doses ${ }^{*} \dagger$} \\
\hline Unvac. & Zero doses & $157287(69.6)$ & ref. \\
\hline \multirow[t]{3}{*}{ Vac. } & One dose & $1937(72.2)$ & 1.05 (1.0 to 1.10$)$ \\
\hline & Two doses & $2883(74.4)$ & $1.08(1.04$ to 1.12$)$ \\
\hline & $\begin{array}{l}\text { Three } \\
\text { doses }\end{array}$ & $21548(74.6)$ & $1.11(1.10$ to 1.13$)$ \\
\hline
\end{tabular}

*HR adjusted for education, income and migration history. †Exposure level of vaccine dose is defined at study entry, as opposed to being a time-varying exposure.

HPV, human papillomavirus; Unvac, unvaccinated;

Vac., HPV vaccinated with $\geq 1$ dose.

\section{Sensitivity analyses}

When redefining subjects as vaccinated after only the full three doses, vaccination uptake was only $11.1 \%$, but results remained unchanged. Results also remained unchanged when excluding the $5.6 \%$ of women who were invited after age 24 , and when redefining vaccination as a time-varying exposure (see online supplementary 4 ).

\section{DISCUSSION}

Young HPV-vaccinated women, most of which were vaccinated under a national benefits scheme, were $32 \%$ more likely to attend cervical screening than unvaccinated women. The difference decreased to $10 \%$ after adjusting for education, income and migration history, suggesting that the crude association is at least partially driven by socioeconomic factors. This is similar to our previous evaluation of attendance in adult HPV-vaccinated women in the same screening programme. ${ }^{18}$

This finding does not support the notion that HPV-vaccinated women might discontinue screening. Any discussion of causal factors behind the observed association must however be cautious due to the observational design. One possible explanation is that vaccination acts as an educational opportunity; raising awareness about cervical cancer and increasing women's propensity to screen. In this cohort, HPV awareness was further amplified by radio, television and letter campaigns targeted at female teenagers, which started in June $2007^{3}$ and perhaps most strongly resonated with vaccinated women. Another possibility is that positive attitude to preventive health services clusters in women who are both vaccinated and attend screening. The overproportionate vaccine uptake among women who initiated screening before being invited also hints in this direction. The clustering of HPV-related and screening-related health behaviours is a known phenomenon, ${ }^{24}{ }^{30}$ implying that the disparity in cervical cancer prevention efforts between the "worried well' ${ }^{31}$ and the unvaccinated non-attenders may be widening also in Sweden.

Our interaction analyses should provide insight into the interplay between the HPV vaccination and socioeconomic factors. The association between vaccination and screening was weakest in wealthy and in Swedish-born women and strongest in low-income and in immigrant women. It should however be noted that vaccine uptake in immigrated women was comparatively low at $1 \%-8 \%$ (data not shown), hence strong self-selection may well have been present. Overall, being vaccinated seems to only be associated with a small attendance increment among wealthier Swedish-born women, while larger increments were observed in low-income groups and those with a migration background.

The findings of this study contribute to a growing body of evidence that the HPV vaccination is not associated with reduced cervical screening uptake. With one exception, numerous empirical studies on vaccinated women's screening participation showed consistent results across 
Table 3 Interaction models on the association between HPV vaccination and cervical screening attendance $(n=261434)$

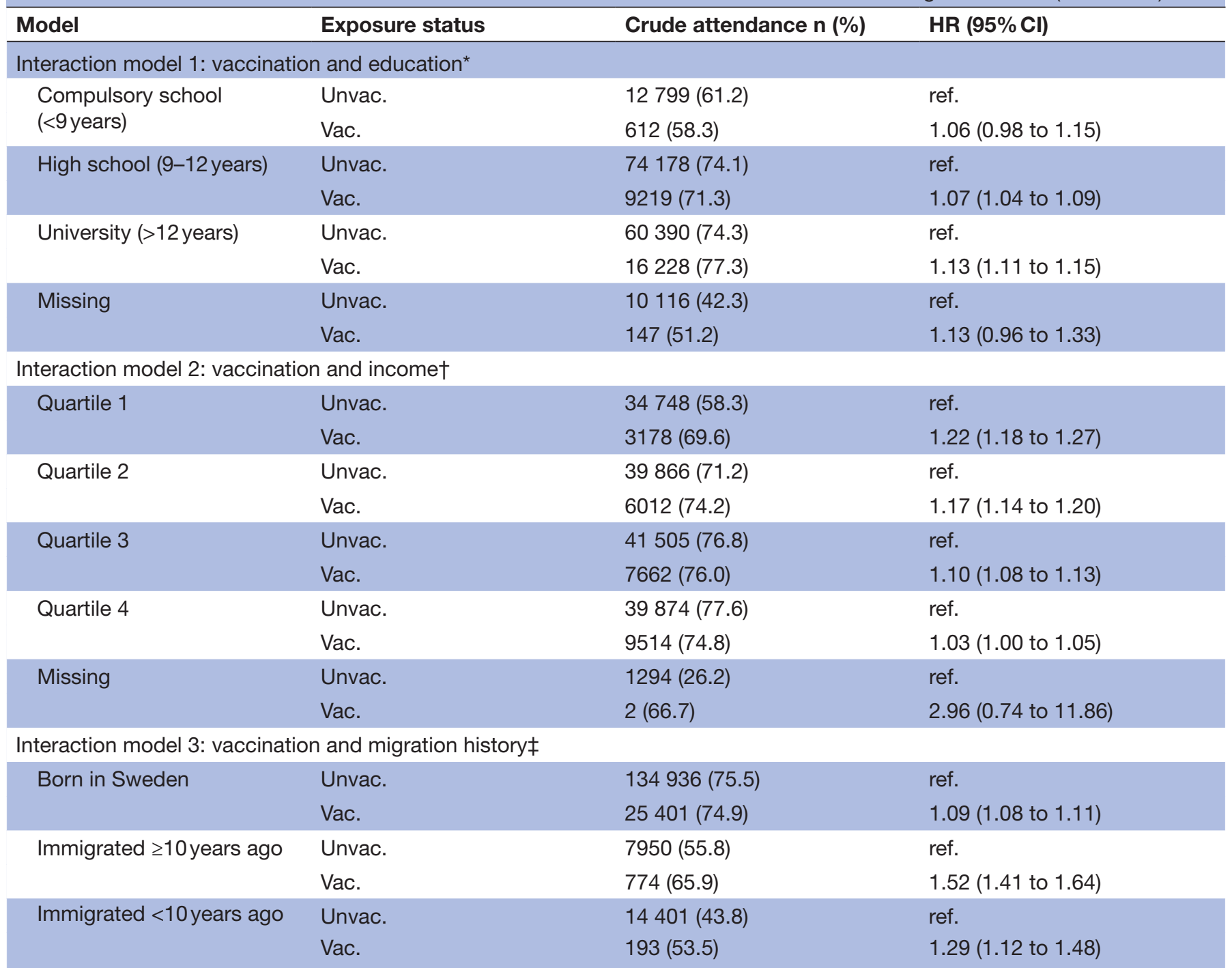

*HR adjusted for income, migration history and an interaction term between vaccination and education. †HR adjusted for education, migration history and an interaction term between vaccination and income. †HR adjusted for income, education and an interaction term between vaccination and migration history. HPV, human papillomavirus; Unvac, unvaccinated; Vac., HPV vaccinated with $\geq 1$ dose.

varied national and social contexts. Register-based Welsh and Scottish studies on catch-up vaccinated cohorts with higher HPV vaccination uptake levels than this study $(49 \%$ and $36 \%)$ and lower overall screening attendance (46\% and $47 \%$ ) also reported higher attendance among the vaccinated. ${ }^{19}{ }^{20}$ Four American studies report similar findings, studying the general population, ${ }^{32}$ members of an integrated healthcare setting ${ }^{24}$ and women in a safety net health system, ${ }^{21}{ }^{23}$ respectively. In contrast, an Australian study reported a $10 \%$ lowered attendance among HPV-vaccinated women, ${ }^{16}$ which might in part have been due to context-specific factors or a differential overestimation of non-attendance due to data mismatching. ${ }^{16}$ Mismatching would not have occurred in the current study due to the individual data linkage.

This study's results closely mirror a Swedish study on the preceding 10 birth cohorts 1977-1987 with $0.8 \%$ vaccine uptake. ${ }^{18}$ The cumulative incidence proportion of screening over 3 years' follow-up time was similar between the preceding 10 cohorts and the study cohorts: $86 \%$ of vaccinated and $75 \%$ of unvaccinated women screened in the preceding cohorts, compared with a respective $85 \%$ and $75 \%$ in the present cohorts. It suggests that the positive association between vaccination and screening is robust, even if vaccine uptake levels increase.

A positive association with screening attendance increased with vaccine dosage number in some studies ${ }^{20}{ }^{24}$ while the opposite pattern was found in an American study. ${ }^{23}$ The present study observed a trend towards higher attendance with more doses, yet CI overlapped. As dose was determined at study entry, some women may have received further doses during follow-up, which were not counted. While the absolute differences 
between doses should thus be interpreted cautiously, the trend appears consistent with most previous studies. ${ }^{21} 2324$

This national cohort study has its major strength in data completeness and the individual linkage to socioeconomic covariates. All registered women from birth years 1988-1991 were followed for the entire study period with minimal attrition. Data on invitations and screening events were complete. Confounding and interactions were treated in detail given the individual data linkage. A further strength is the prospective objective data collection, which eliminates the possibility of recall bias. Hence, the overall level of differential misclassification and bias in this study should be minimal and the internal validity should be high.

The main limitation of this observational study is that the participants still were not part of an organised schoolbased programme, and thus self-selected (on their own and/or parental volition) whether to be vaccinated or not. Despite excluding the most highly self-selected subgroup who screened early on their own initiative, socioeconomic selection mechanisms likely determined the choice to be vaccinated given the vaccine's substantial cost. This limitation was addressed by adjusting for socioeconomic factors, yet some residual confounding could remain and selection bias is likely still present.

A second limitation is the $85 \%-90 \%$ completeness of the SVEVAC vaccination register compared with number of sold doses in the nation, ${ }^{5}$ due to which some vaccinations were missed even after complementing with PDR data. Moreover, vaccinated immigrant women or native Swedes who may have vaccinated abroad would have been misclassified as unvaccinated. Yet, given that vaccinated women were more likely to attend screening, erroneously classifying them as unvaccinated would, if anything, have biased results towards the null hypothesis by reducing between-group differences. The observed association between vaccination and screening should thus not be overestimated due to such misclassification.

Future research needs to be repeated in routinely HPV-immunised women such as the Swedish birth cohorts 1993-1998. These women were offered free catch-up vaccinations at ages $13-18$ and reached $60 \%-80 \%$ vaccine uptake; girls born in 1999 and later were offered free school-based vaccinations at ages 10-12 and reached $85 \%$ uptake. ${ }^{5}$ These younger girls will be affected by less HPV-related diseases and lower perceived infection risk due to the vaccination, ${ }^{917}$ which may alter the association between vaccination and screening.

The implications of this study are twofold. First, there is currently no apparent need for targeted education campaigns to the HPV-vaccinated women under study, as they have an equal or higher cervical screening uptake. Second, the advent of HPV vaccination seems to widen the disparity between the risk groups for cervical lesions and cancer. Women who are neither vaccinated nor attend screening regularly face a double vulnerability. ${ }^{243133}$ A similarly stronger association between vaccination and screening attendance among the more deprived has also been observed in Scotland, where register-based studies similar to this are also readily feasible. ${ }^{20}$ These findings imply an even greater disparity of cervical cancer risk among deprived girls and young women that warrants particular concern and intervention. Continuous monitoring of attendance rates in later birth cohorts eligible for free-of-charge school-based vaccination, which achieved higher vaccine uptake and lower socioeconomic disparity, ${ }^{34}$ will be key to inform this ongoing issue. Future prevention efforts should consider how to better reach this subgroup.

\section{CONCLUSIONS}

This nationwide study found that HPV vaccination is associated with higher cervical screening attendance among Swedish girls and women born in 1988-1991. Future studies should continue to assess the association between HPV vaccination and screening in younger, routinely vaccinated cohorts.

Acknowledgements The authors thank Pouran Almstedt for data management, and Jiayao Lei and Eva Herweijer for statistical input.

Contributors TK: conception and design of the work, data analysis, data interpretation, drafting of the manuscript, critical revision of the article, approval of final draft. JW: data interpretation, critical revision of the article, approval of final draft. PS: conception and design of the work, data interpretation, critical revision of the article, approval of final draft. KS: conception and design of the work, data collection, data analysis, data interpretation, critical revision of the article, approval of final draft.

Funding This study was supported by the Swedish Foundation for Strategic Research (grant number KF10-0046) and by the Nordic Information for Action eScience Center (NIASC), a Nordic Center of Excellence in eScience funded by NordForsk (Project no. 62721).

Disclaimer The study sponsor did not participate in study design, data collection, analysis, interpretation of data, writing of the article or the decision to submit it for publication.

Competing interests KS has received grants from MSD for other studies on human papillomavirus vaccination in Sweden. No industry funding was used for the current work.

\section{Patient consent Not required.}

Ethics approval Ethical approval was obtained from the Regional Ethical Review Board in Stockholm (Dnr 2006/1053-31/2 and EPN Dnr 2012/216-32), which determined that informed consent was not required due to the study's populationbased nature.

Provenance and peer review Not commissioned; externally peer reviewed.

Data sharing statement All relevant source data are shown in the manuscript and supplementary files. If access to raw data is required, please contact par.sparen@ ki.se. Data may be shared if all ethical and legal requirements are met for such a request.

Open access This is an open access article distributed in accordance with the Creative Commons Attribution Non Commercial (CC BY-NC 4.0) license, which permits others to distribute, remix, adapt, build upon this work non-commercially, and license their derivative works on different terms, provided the original work is properly cited, appropriate credit is given, any changes made indicated, and the use is non-commercial. See: http://creativecommons.org/licenses/by-nc/4.0/.

\section{REFERENCES}

1. de Freitas AC, Gurgel AP, Chagas BS, et al. Susceptibility to cervical cancer: an overview. Gynecol Oncol 2012;126:304-11. 
2. Chesson HW, Dunne EF, Hariri S, et al. The estimated lifetime probability of acquiring human papillomavirus in the United States. Sex Transm Dis 2014;41:660-4.

3. Sundström K, Tran TN, Lundholm C, et al. Acceptability of HPV vaccination among young adults aged 18-30 years--a population based survey in Sweden. Vaccine 2010;28:7492-500.

4. Tandvårds och Läkemedelsförmånsverket (TLV). Decisions on pricing of HPV vaccines. https://www.tlv.se (accessed 9 May 2017).

5. Public Health Agency of Sweden, 2017. Statistic for HPV vaccinations https://www.folkhalsomyndigheten.se/globalassets/ smittskydd-sjukdomar/vaccinationer/statistik-for-hpv-vaccinationerandel-vaccinerade-flickor-tom-2015-12-31.pdf (accessed 9 May 2017).

6. Collins S, Mazloomzadeh S, Winter $\mathrm{H}$, et al. High incidence of cervical human papillomavirus infection in women during their first sexual relationship. BJOG 2002;109:96-8.

7. Hildesheim A, Herrero R, Wacholder S, et al. Effect of human papillomavirus 16/18 L1 viruslike particle vaccine among young women with preexisting infection: a randomized trial. JAMA 2007;298:743-53.

8. Block SL, Nolan T, Sattler C, et al. Comparison of the immunogenicity and reactogenicity of a prophylactic quadrivalent human papillomavirus (types 6,11, 16, and 18) L1 virus-like particle vaccine in male and female adolescents and young adult women. Pediatrics 2006;118:2135-45.

9. Herweijer E, Sundström K, Ploner A, et al. Quadrivalent HPV vaccine effectiveness against high-grade cervical lesions by age at vaccination: a population-based study. Int J Cancer 2016;138:2867-74.

10. Herweijer E, Sundström K, Ploner A, et al. Erratum. Int J Cancer 2017;141:E1-4.

11. Dillner J. Nationellt Kvalitetsregister för Cervixcancerprevention (NKCx) Verksamhetsberättelse och Årsrapport 2016, 2016

12. Andrae B, Kemetli L, Sparén P, et al. Screening-preventable cervical cancer risks: evidence from a nationwide audit in Sweden. J Natl Cancer Inst 2008;100:622-9.

13. WHO. The global advisory committee on vaccine safety. Committee report 11-12. Geneva: WHO, 2014.

14. Cooper Robbins SC, Bernard D, McCaffery K, et al. "Is cancer contagious?": Australian adolescent girls and their parents: making the most of limited information about HPV and HPV vaccination. Vaccine 2010;28:3398-408.

15. Kulasingam SL, Pagliusi S, Myers E. Potential effects of decreased cervical cancer screening participation after HPV vaccination: an example from the U.S. Vaccine $2007 ; 25: 8110-3$

16. Budd AC, Brotherton JM, Gertig DM, et al. Cervical screening rates for women vaccinated against human papillomavirus. Med J Aust 2014;201:279-82.

17. Baldur-Felskov B, Dehlendorff C, Munk C, et al. Early impact of human papillomavirus vaccination on cervical neoplasia-nationwide follow-up of young Danish women. J Natl Cancer Inst 2014;106:djt460
18. Herweijer E, Feldman AL, Ploner A, et al. The participation of HPV-vaccinated women in a national cervical screening program: population-based cohort study. PLoS One 2015;10:e0134185-12.

19. Beer $\mathrm{H}$, Hibbitts S, Brophy S, et al. Does the HPV vaccination programme have implications for cervical screening programmes in the UK? Vaccine 2014;32:1828-33.

20. Palmer TJ, McFadden M, Pollock KG et al. HPV immunisation and increased uptake of cervical screening in Scottish women; observational study of routinely collected national data. $\mathrm{Br} J$ Cancer 2016;114:576-81.

21. Paynter CA, Van Treeck BJ, Verdenius I, et al. Adherence to cervical cancer screening varies by human papillomavirus vaccination status in a high-risk population. Prev Med Rep 2015;2:711-6.

22. Sauer AG, Jemal A, Simard EP, et al. Differential uptake of recent Papanicolaou testing by HPV vaccination status among young women in the United States, 2008-2013. Cancer Epidemiol 2015;39:650-5.

23. Boone SD, Pinkston CM, Baumgartner KB, et al. Associations between prior HPV4 vaccine doses and cervical cancer screening participation. Cancer Epidemiol 2016;42:108-14.

24. Chao C, Silverberg MJ, Becerra TA, et al. Human papillomavirus vaccination and subsequent cervical cancer screening in a large integrated healthcare system. Am J Obstet Gynecol 2017;216:151. e1-151.e9.

25. Magnusson PK, Lichtenstein P, Gyllensten UB. Heritability of cervical tumours. Int J Cancer 2000;88:698-701.

26. The National Board of Health and Welfare, 2017. Information on the prescribed drug register http://www.socialstyrelsen.se/register/ halsodataregister/lakemedelsregistret (accessed 9 May 2017).

27. Statistics Sweden. Longitudinell integrationsdatabas för Sjukförsäkrings- och Arbetsmarknadsstudier (LISA) 1990-2013, 2013.

28. Leval A, Herweijer E, Ploner A, et al. Quadrivalent human papillomavirus vaccine effectiveness: a Swedish national cohort study. J Natl Cancer Inst 2013;105:469-74.

29. Schoenfeld D. Partial residuals for the proportional hazards regression model. Biometrika 1982;69:51:239-41.

30. Kasting ML, Shapiro GK, Rosberger Z, et al. Tempest in a teapot: A systematic review of HPV vaccination and risk compensation research. Hum Vaccin Immunother 2016;12:1435-50.

31. Harper DM, DeMars LR. HPV vaccines - a review of the first decade. Gynecol Oncol 2017;146:196-204.

32. Kim J, Bell C, Sun M, et al. Effect of human papillomavirus vaccination on cervical cancer screening in Alberta. CMAJ 2016;188:E281-8.

33. Hirth JM, Lin YL, Kuo YF, et al. Effect of number of human papillomavirus vaccine doses on guideline adherent cervical cytology screening among 19-26year old females. Prev Med 2016;88:134-9.

34. Wang J, Sparén P, Netterlid E, et al. Demographic and socioeconomic determinants of HPV vaccine uptake in Sweden [abstract]. Eurogin 2016 Abstracts, Part I - Main Conference Program 2016:273-4. 